\title{
DYNAMIC STABILITY OF EQUILIBRIUM STATES OF THE EXTENSIBLE BEAM ${ }^{1}$
}

\author{
R. W. DICKEY ${ }^{2}$
}

ABSTRACT. In this paper an equation describing the dynamic behavior of a nonlinear beam with viscous damping is treated. In particular it is shown that when the trivial solution is the only equilibrium solution then all solutions, regardless of intial data, decay exponentially to the trivial solution. In those cases where nontrivial equilibrium solutions in addition to the trivial solution are possible it is shown that the nontrivial solution corresponding to the "lowest buckled mode' is locally stable, i.e. dynamic solutions with initial data 'close' to the lowest buckled mode decay to this equilibrium solution. Estimates are obtained for the various decay rates.

1. Introduction. The purpose of this paper is to discuss the asymptotic stability of equilibrium solutions of the equation

$$
w_{t t}+\alpha w_{t}+w_{x x x x}-\left[(-1+\varepsilon)+\frac{2}{\pi} \int_{0}^{\pi} w_{x}^{2} d x\right] w_{x x}=0 \quad(\alpha>0)
$$

which satisfy initial data

$$
\begin{aligned}
w(x, 0) & =f(x), \\
w_{t}(x, 0) & =g(x)
\end{aligned}
$$

and boundary conditions

$$
\begin{aligned}
w(0, t) & =w(\pi, t)=0, \\
w_{x x}(0, t) & =w_{x x}(\pi, t)=0 .
\end{aligned}
$$

Solutions of (1.1) describe the damped vibrations of an extensible beam (cf. [1]).

The existence of solutions to equation (1.1) with $\alpha=0$ has been treated in [2] and [3] and there is no difficulty in extending these arguments to prove the existence of solutions to (1.1) with $\alpha>0$ for all $t \geqq 0$. In this paper it will be assumed that the initial data (1.2) is sufficiently differentiable

Received by the editors October 18, 1972 and, in revised form, February 15, 1973.

AMS (MOS) subject classifications (1970). Primary 34A35; Secondary 35L60.

${ }^{1}$ This research was supported by the National Science Foundation, contract No. GP-28699 and the Wisconsin Alumni Research Foundation.

${ }^{2}$ This research was done while the author was an SRC Senior Visiting Fellow at the University of Newcastle upon Tyne, Newcastle upon Tyne, England.

(c) American Mathematical Society 1973 
to guarantee that solutions of (1.1) are classical solutions. In this case it is quite easily shown following the development in [2] that the solution can be written in the form

$$
w(x, t)=\sum_{j=1}^{\infty} T_{j}(t) \sin j x
$$

where the functions $T_{j}(t)$ exist for all $t \geqq 0$ and satisfy the infinite system of ordinary differential equations

$$
\ddot{T}_{j}+\alpha \dot{T}_{j}+j^{4} T_{j}+j^{2}\left[(-1+\varepsilon)+\sum_{l=1}^{\infty} l^{2} T_{l}^{2}\right] T_{j}=0
$$

$(\cdot=d / d t)$ and the initial conditions

$$
\begin{gathered}
-T_{j}(0)=\alpha_{j}=\frac{2}{\pi} \int_{0}^{\pi} f(x) \sin j x d x, \\
\dot{T}_{j}(0)=\beta_{j}=\frac{2}{\pi} \int_{0}^{\pi} g(x) \sin j x d x .
\end{gathered}
$$

If $\varepsilon \geqq 0$ the 'static' problem corresponding to (1.1), i.e.

$$
W_{x x x x}-\left[(-1+\varepsilon)+\frac{2}{\pi} \int_{0}^{\pi} W_{x}^{2} d x\right] W_{x x}=0
$$

has only the trivial solution satisfying the boundary conditions (1.3). In this case (cf. \$2) it will be shown that $w \equiv 0$ is globally stable, i.e. every solution $w(x, t)$ of $(1.1)$ has the property that $w(x, t) \rightarrow 0$ as $t \rightarrow \infty$. If $\varepsilon<0$ equation (1.7) has, in addition to the trivial solution, solutions of the form

$$
W_{j}^{ \pm}(x)= \pm \frac{1}{j} \sqrt{1-\varepsilon-j^{2}} \sin j x, \quad j=1,2, \cdots, N,
$$

where $N$ is the largest integer such that $N^{2}<1-\varepsilon$. In $\S 3$ conditions on the initial data are given which guarantee that if $\varepsilon<0$ then $w(x, t) \rightarrow 0$ as $t \rightarrow \infty$. This result is actually sufficient to guarantee that $w(x, t)$ converges to one of the buckled states $W_{j}^{ \pm}(x)$ (cf. [4]). Finally, in $\S 4$ it is shown that if the initial data is sufficiently 'close' to $W_{1}^{+}(x)\left(W_{1}^{-}(x)\right)$, then $w(x, t) \rightarrow$ $W_{1}^{+}(x)\left(w(x, t) \rightarrow W_{1}^{-}(x)\right)$ as $t \rightarrow \infty$, i.e. if $\varepsilon<0$ then the lowest buckled mode is 'locally' stable. The above results were obtained formally, using two time asymptotic expansions, by Reiss and Matkowsky [6].

2. Stability of the trivial solution. In this section it will be shown that if $\varepsilon \geqq 0$ solutions of (1.1) tend to zero as $t \rightarrow \infty$. This result is based on the fact that solutions of (1.1) satisfying the boundary conditions (1.3) must 
satisfy the identities

$$
\begin{aligned}
\frac{d E}{d t}+ & 2 \alpha \int_{0}^{\pi} w_{t}^{2} d x \\
= & \frac{d}{d t}\left\{\int_{0}^{\pi} w_{t}^{2} d x+\int_{0}^{\pi} w_{x x}^{2} d x+(\varepsilon-1) \int_{0}^{\pi} w_{x}^{2} d x+\frac{1}{\pi}\left(\int_{0}^{\pi} w_{x}^{2} d x\right)^{2}\right\} \\
& +2 \alpha \int_{0}^{\pi} w_{t}^{2} d x=0
\end{aligned}
$$

and

(2.2) $\frac{d}{d t} \int_{0}^{\pi} w w_{t} d x-2 \int_{0}^{\pi} w_{t}^{2} d x+E+\frac{1}{\pi}\left(\int_{0}^{\pi} w_{x}^{2} d x\right)^{2}+\alpha \int_{0}^{\pi} w w_{t} d x=0$.

The identities (2.1) and (2.2) follow upon multiplying equation (1.1) first by $w_{t}$ and then by $w$. Multiplying equation (2.2) by $\alpha$ and adding the result to (2.1) yields the identity ${ }^{3}$

$$
\frac{d}{d t}\left\{E+\alpha \int_{0}^{\pi} w w_{t} d x\right\}+\alpha\left\{E+\alpha \int_{0}^{\pi} w w_{t} d x\right\}=-\frac{\alpha}{\pi}\left(\int_{0}^{\pi} w_{x}^{2} d x\right)^{2} \leqq 0
$$

or

$$
E(t)+\alpha \int_{0}^{\pi} w w_{t} d x \leqq\left(E(0)+\alpha \int_{0}^{\pi} f(x) g(x) d x\right) \exp (-\alpha t)
$$

where

$$
\begin{aligned}
E(0)= & \int_{0}^{\pi} g(x)^{2} d x+\int_{0}^{\pi} f^{\prime \prime}(x)^{2} d x \\
& +(\varepsilon-1) \int_{0}^{\pi} f^{\prime}(x)^{2} d x+\frac{1}{\pi}\left(\int_{0}^{\pi} f^{\prime}(x)^{2} d x\right)^{2} .
\end{aligned}
$$

The functions $w(x, t)$ and $w_{x x}(x, t)$ vanish at $x=0$ and $x=\pi$. Thus the minimum characterization of eigenvalues (cf. [5]) guarantees that

$$
\int_{0}^{\pi} w_{x x}^{2} d x / \int_{0}^{\pi} w_{x}^{2} d x \geqq 1
$$

and

$$
\int_{0}^{\pi} w_{x}^{2} d x / \int_{0}^{\pi} w^{2} d x \geqq 1
$$

since $\lambda=1$ is the smallest eigenvalue of both

$$
\begin{aligned}
w_{x x x x}+\lambda w_{x x} & =0, \\
w_{x x}+\lambda w & =0
\end{aligned}
$$

${ }^{3}$ The identity (2.3) was suggested to the author by P. R. Rabinowitz. 
under the prescribed boundary conditions. We conclude that solutions of (1.1) satisfying the boundary conditions (1.3) must also satisfy the inequality

$$
\int_{0}^{\pi} w^{2} d x \leqq \int_{0}^{\pi} w_{x}^{2} d x \leqq \int_{0}^{\pi} w_{x x}^{2} d x .
$$

This fact in conjunction with (2.4) implies that $w(x, t)$ must satisfy

$$
\begin{aligned}
& \frac{\alpha}{2} \frac{d}{d t} \int_{0}^{\pi} w^{2} d x+\varepsilon \int_{0}^{\pi} w^{2} d x+\frac{1}{\pi}\left(\int_{0}^{\pi} w^{2} d x\right)^{2} \\
& \leqq\left(E(0)+\alpha \int_{0}^{\pi} f(x) g(x) d x\right) \exp (-\alpha t)
\end{aligned}
$$

and in addition the weaker inequality

$$
\frac{\alpha}{2} \frac{d}{d t} \int_{0}^{\pi} w^{2} d x+\varepsilon \int_{0}^{\pi} w^{2} d x \leqq\left(E(0)+\alpha \int_{0}^{\pi} f(x) g(x) d x\right) \exp (-\alpha t) .
$$

If $\varepsilon>0$ the inequality (2.12) implies that either

$$
\int_{0}^{\pi} w^{2} d x \leqq C_{1} \exp (-\alpha t)+C_{2} \exp (-2 \varepsilon t / \alpha)
$$

if $\alpha^{2} \neq 2 \varepsilon$ or

$$
\int_{0}^{\pi} w^{2} d x \leqq\left(C_{3}+C_{4} t\right) \exp (-\alpha t)
$$

if $\alpha^{2}=2 \varepsilon$. The constants $C_{1}, C_{2}, C_{3}$ and $C_{4}$ depend only on the initial data. In either case (2.12) and (2.13) imply that $w(x, t) \rightarrow 0$ as $t \rightarrow \infty$ if $\varepsilon>0$. If $\varepsilon=0$ it is easily shown, using (2.11), that

$$
\frac{d}{d t}\left(\int_{0}^{\pi} w^{2} d x\right)^{2} \leqq C_{5}
$$

and

$$
\int_{0}^{t}\left(\int_{0}^{\pi} w^{2} d x\right)^{2} d \tau \leqq C_{6}
$$

where $C_{5}$ and $C_{6}$ depend on the initial data. It is an immediate consequence of (2.15) and (2.16) that, even in the case $\varepsilon=0, w(x, t) \rightarrow 0$ as $t \rightarrow \infty$. We summarize these results in the following theorem:

THEOREM (2.1). If $\varepsilon \geqq 0$ every solution $w(x, t)$ of (1.1) satisfying the boundary conditions (1.3) has the property that $w(x, t) \rightarrow 0$ as $t \rightarrow \infty$.

3. Conditions for which $w(x, t) \nrightarrow 0$ as $t \rightarrow \infty$. If $\varepsilon<0$ it is possible to specify initial data such that $E(0)<0$. For example, if

$$
w(x, 0)=W_{1}^{\llcorner}(x)= \pm \sqrt{-\varepsilon} \sin x
$$


and $w_{t}(x, 0)=0$ it is easily seen that $E(0)=-\pi \varepsilon^{2} / 4$. In this section it will be shown that:

THEOREM (3.1). Every solution $w(x, t)$ of (1.1) satisfying the boundary conditions (1.3) and the condition $E(0)<0$ has the property and $w(x, t) \rightarrow 0$ as $t \rightarrow \infty$.

Proof. If $E(0)<0(\Rightarrow \varepsilon<0)$ the identity $(2.1)$ implies that

$$
E(t) \leqq E(0)<0
$$

for all $t \geqq 0$. In view of (2.10) the inequality (3.1) yields

$$
\varepsilon \pi \int_{0}^{\pi} w_{x}^{2} d x+\left(\int_{0}^{\pi} w_{x}^{2} d x\right)^{2}-\pi E(0) \leqq 0
$$

or equivalently

$$
0<M_{-} \leqq \int_{0}^{\pi} w_{x}^{2} d x \leqq M_{1}
$$

where

$$
M_{ \pm}=\frac{\pi}{2}\left\{-\varepsilon \pm\left(\varepsilon^{2}+4 E(0) / \pi\right)^{1 / 2}\right\} .
$$

(Note that (3.3) and (3.4) imply that $E(0) \geqq-\pi \varepsilon^{2} / 4$, i.e. the minimum energy is attained when $w(x, t)=W_{1}(x)$.) It is an immediate consequence of (3.1) and (3.3) that there exists a positive constant $M$ such that

$$
\int_{0}^{\pi} w_{x x}^{2} d x \leqq M
$$

for all $t \geqq 0$. In addition the inequality (3.3) suffices to guarantee that $w_{x}(x, t) \nrightarrow 0$ as $t \rightarrow \infty$. It remains to show that $w(x, t) \nrightarrow 0$ as $t \rightarrow \infty$. However, this follows from the fact that

$$
\int_{0}^{\pi} w_{x}^{2} d x=-\int_{0}^{\pi} w w_{x x} d x \leqq\left(\int_{0}^{\pi} w^{2} d x \int_{0}^{\pi} w_{x x}^{2} d x\right)^{1 / 2} .
$$

The inequalities (3.3), (3.5), and (3.6) imply that

$$
\int_{0}^{\pi} w^{2} d x \geqq \frac{M^{2}}{M}>0 .
$$

Thus, $w(x, t) \rightarrow 0$ as $t \rightarrow \infty$. Q.E.D.

4. Local stability of the lowest buckled mode. The equilibrium solutions $W_{1}^{\llcorner}(x)$ of (1.1) are not globally stable even though $\varepsilon<0$. Indeed when $\varepsilon<0$ there exist solutions of (1.1) which tend asymptotically to each of the 
possible equilibrium solutions (cf. [6]). What is true and will be shown in this section is that solutions which 'start' sufficiently close to $W_{1}^{ \pm}(x)$ decay to $W_{1}^{ \pm}(x)$ as $t \rightarrow \infty$.

In order to show that $W_{1}^{+}(x)$ is locally stable when $\varepsilon<0$ it is convenient to adopt the Fourier series representation of the solution (cf. (1.4)). In this case the identity (2.3) becomes

$$
\frac{d}{d t}\left\{E\left(T_{j}\right)+\alpha \sum_{j=1}^{\infty} T_{j} \dot{T}_{j}\right\}+\alpha\left\{E\left(T_{j}\right)+\alpha \sum_{j=1}^{\infty} T_{j} \dot{T}_{j}+\frac{1}{2}\left(\sum_{j=1}^{\infty} j^{2} T_{j}^{2}\right)^{2}\right\}=0
$$

where

$$
E\left(T_{j}\right)=\sum_{j=1}^{\infty} \dot{T}_{j}^{2}+\sum_{j=1}^{\infty} j^{4} T_{j}^{2}+(\varepsilon-1) \sum_{j=1}^{\infty} j^{2} T_{j}^{2}+\frac{1}{2}\left(\sum_{j=1}^{\infty} j^{2} T_{j}^{2}\right)^{2} .
$$

In order to show that $w(x, t) \rightarrow W_{1}^{+}(x)$ it suffices to show that $T_{1}(t) \rightarrow \sqrt{-\varepsilon}$ and $T_{j}(t) \rightarrow 0$ for $j=2,3, \cdots$ as $t \rightarrow \infty$. For this purpose define new variables

$$
\begin{aligned}
& S_{1}=T_{1}-\sqrt{-\varepsilon}, \\
& S_{j}=T_{j}, \quad j=2,3 \cdots .
\end{aligned}
$$

The function $S_{1}$ satisfies the differential equation

$$
\ddot{S}_{1}+\alpha \dot{S}_{1}-2 \varepsilon S_{1}+S_{1}^{2}\left(S_{1}+3 \sqrt{-\varepsilon}\right)+\left(S_{1}+\sqrt{-\varepsilon}\right) \sum_{j=1}^{\alpha_{2}} j^{2} S_{j}^{2}=0 .
$$

The object is to determine conditions on the initial data which guarantee that $S_{j}(t) \rightarrow 0$ as $t \rightarrow \infty$. The identity (4.1) can be rewritten in terms of the functions $S_{j}$ and in fact after some elementary manipulations (4.1) becomes

$$
\begin{aligned}
& \frac{d}{d t}\left\{E\left(S_{j}\right)+\alpha \sum_{j=1}^{\infty} S_{j} \dot{S}_{j}+\alpha \sqrt{-\varepsilon} \dot{S}_{1}\right. \\
& \left.\quad+S_{1}^{2}\left(2 \sqrt{-\varepsilon} S_{1}-3 \varepsilon\right)+\left(2 \sqrt{-\varepsilon} S_{1}-\varepsilon\right) \sum_{j=0}^{\infty} j^{2} S_{j}^{2}\right\} \\
& (4.5)+\alpha\left\{E\left(S_{j}\right)+\alpha \sum_{j=1}^{\infty} S_{j} \dot{S}_{j}+\frac{1}{2}\left(\sum_{j=1}^{\infty} j^{2} S_{j}^{2}\right)^{2}+\alpha \sqrt{-\varepsilon} \dot{S}_{1}-2 \varepsilon \sqrt{-\varepsilon} S_{1}\right. \\
& \left.+S_{1}^{2}\left(4 \sqrt{-\varepsilon} S_{1}-6 \varepsilon\right)+\left(4 \sqrt{-\varepsilon} S_{1}-2 \varepsilon\right) \sum_{j=2}^{\infty} j^{2} S_{j}^{2}\right\}=0
\end{aligned}
$$

or equivalently (cf. (4.4))

$$
\begin{aligned}
& \frac{d}{d t}\left\{E\left(S_{j}\right)+\alpha \sum_{j=1}^{\infty} S_{j} \dot{S}_{j}+S_{1}^{2}\left(2 \sqrt{-\varepsilon} S_{1}-3 \varepsilon\right)\right.\left.+\left(2 \sqrt{-\varepsilon} S_{1}-\varepsilon\right) \sum_{j=2}^{\infty} j^{2} S_{j}^{2}\right\} \\
&(4.6)+\alpha\left\{E\left(S_{j}\right)+\alpha \sum_{j=1}^{\infty} S_{j} \dot{S}_{j}+\frac{1}{2}\left(\sum_{j=1}^{\infty} j^{2} S_{j}^{2}\right)^{2}+S_{1}^{2}\left(3 \sqrt{-\varepsilon} S_{1}-3 \varepsilon\right)\right. \\
&\left.+\left(3 \sqrt{-\varepsilon} S_{1}-\varepsilon\right) \sum_{j=2}^{\infty} j^{2} S_{j}^{2}\right\}=0 .
\end{aligned}
$$


Noting that

$$
\frac{1}{2} \sum_{j=1}^{\infty} \dot{S}_{j}^{2}+\alpha \sum_{j=1}^{\infty} S_{j} \dot{S}_{j}=\frac{1}{2} \sum_{j=1}^{\infty}\left(\dot{S}_{j}+\alpha S_{j}\right)^{2}-\frac{\alpha^{2}}{2} \sum_{j=1}^{\infty} S_{j}^{2}
$$

the identity (4.6) can be rewritten

$$
\frac{d}{d t} P+\alpha Q=-\frac{1}{2}\left(\sum_{j=1}^{\infty} j^{2} S_{j}^{2}\right) \leqq 0
$$

where

$$
\begin{aligned}
P= & \frac{1}{2} \sum_{j=1}^{\infty} \dot{S}_{j}^{2}+\frac{1}{2} \sum_{j=1}^{\infty}\left(\dot{S}_{j}+\alpha S_{j}\right)^{2}+\sum_{j=1}^{\infty} j^{4} S_{j}^{2}+(\varepsilon-1) \sum_{j=1}^{\infty} j^{2} S_{j}^{2} \\
& +\frac{1}{2}\left(\sum_{j=1}^{\infty} j^{2} S_{j}^{2}\right)^{2}+S_{1}^{2}\left(2 \sqrt{-\varepsilon} S_{1}-3 \varepsilon\right)+\left(2 \sqrt{-\varepsilon} S_{1}-\varepsilon\right) \sum_{j=2}^{\infty} j^{2} S_{j}^{2}, \\
Q= & \sum_{j=1}^{\infty} \dot{S}_{j}^{2}+\sum_{j=1}^{\infty} j^{4} S_{j}^{2}+(\varepsilon-1) \sum_{j=1}^{\infty} j^{2} S_{j}^{2}+\frac{1}{2}\left(\sum_{j=1}^{\infty} j^{2} S_{j}^{2}\right)^{2} \\
& +S_{1}^{2}\left(3 \sqrt{-\varepsilon} S_{1}-3 \varepsilon\right)+\left(3 \sqrt{-\varepsilon} S_{1}-\varepsilon\right) \sum_{j=2}^{\infty} j^{2} S_{j}^{2} .
\end{aligned}
$$

Both $P$ and $Q$ are nonnegative if $\left|S_{1}\right|$ is sufficiently small. In order to see this, simply note that

$$
\begin{aligned}
P= & \frac{1}{2} \sum_{j=1}^{\infty} \dot{S}_{j}^{2}+\frac{1}{2} \sum_{j=1}^{\infty}\left(\dot{S}_{j}+\alpha S_{j}\right)^{2}+\frac{1}{2}\left(\sum_{j=1}^{\infty} j^{2} S_{j}^{2}\right)^{2} \\
& +S_{1}^{2}\left(2 \sqrt{-\varepsilon} S_{1}-2 \varepsilon\right)+\sum_{j=2}^{\infty}\left(2 \sqrt{-\varepsilon} S_{1}+j^{2}-1\right) j^{2} S_{j}^{2}, \\
Q= & \sum_{j=1}^{\infty} \dot{S}_{j}^{2}+\frac{1}{2}\left(\sum_{j=1}^{\infty} j^{2} S_{j}^{2}\right)^{2} \\
& +S_{1}^{2}\left(3 \sqrt{-\varepsilon} S_{1}-2 \varepsilon\right)+\sum_{j=2}^{\infty}\left(3 \sqrt{-\varepsilon} S_{1}+j^{2}-1\right) j^{2} S_{j}^{2}
\end{aligned}
$$

and thus both $P$ and $Q$ are nonnegative if

$$
\left|S_{1}\right| \leqq \min (2 \sqrt{-\varepsilon} / 3,1 / \sqrt{-\varepsilon})=\gamma .
$$

The next step in the procedure is to estimate $P$ in terms of $Q$ while assuming $\left|S_{1}\right|$ satisfies the bound (4.11). For this purpose note that since

$$
\alpha \sum_{j=1}^{\infty} S_{j} \dot{S}_{j} \leqq \frac{1}{2} \sum_{j=1}^{\infty} \dot{S}_{j}^{2}+\frac{\alpha^{2}}{2} \sum_{j=1}^{\infty} S_{j}^{2}
$$


$P$ can be estimated by

$$
\begin{aligned}
P \leqq & \frac{3}{2} \sum_{j=1}^{\infty} \dot{S}_{j}^{2}+\frac{1}{2}\left(\sum_{j=1}^{\infty} j^{2} S_{j}^{2}\right)^{2}+S_{1}^{2}\left(2 \sqrt{-\varepsilon} S_{1}-2 \varepsilon+\alpha^{2}\right) \\
& +\sum_{j=2}^{\infty}\left(2 \sqrt{-\varepsilon} S_{1}+j^{2}-1+\alpha^{2} / j^{2}\right) j^{2} S_{j}^{2} .
\end{aligned}
$$

If $\left|S_{1}\right| \leqq \gamma-\delta \leqq 2 \sqrt{-\varepsilon} / 3-\delta(\delta>0)$ then

$$
\left(2 \sqrt{-\varepsilon} S_{1}-2 \varepsilon+\alpha^{2}\right) \leqq C_{1}\left(3 \sqrt{-\varepsilon} S_{1}-2 \varepsilon\right)
$$

if

$$
C_{1}=\left(2 \delta \sqrt{-\varepsilon}-2 \varepsilon / 3+\alpha^{2}\right) /(3 \delta \sqrt{-\varepsilon})
$$

and if $\left|S_{1}\right| \leqq \gamma-\delta \leqq 1 / \sqrt{-\varepsilon}-\delta(\delta>0)$ then

$$
\left(2 \sqrt{-\varepsilon} S_{1}+j^{2}-1+\alpha^{2} / j^{2}\right) \leqq C_{2}\left(3 \sqrt{-\varepsilon} S_{1}+j^{2}-1\right)
$$

$$
j=2,3, \cdots \text {. }
$$

where

$$
C_{2}=\max _{j=2,3, \cdots} \frac{2 \delta \sqrt{-\varepsilon}+j^{2}-3+\alpha^{2} / j^{2}}{3 \delta \sqrt{-\varepsilon}+j^{2}-4} .
$$

Combining this result with (4.12) yields

$$
P \leqq \beta Q
$$

where

$$
\beta=\max \left(3 / 2, C_{1}, C_{2}\right)
$$

if $\left|S_{1}\right| \leqq \gamma-\delta$. Thus (4.8) implies that

$$
d P / d t+\alpha P / \beta \leqq 0
$$

or

$$
P(t) \leqq P(0) \exp (-\alpha t / \beta)
$$

as long as $\left|S_{1}\right| \leqq \gamma-\delta$. Assume that

$$
P(0) \leqq(\gamma-\delta)^{4} / 2
$$

and

$$
\left|S_{1}(0)\right|<\gamma-\delta .
$$

In this case $\left|S_{1}(t)\right|<\gamma-\delta$ in some interval. Assume there exists a value $t=t^{*}$ such that $\left|S_{1}\left(t^{*}\right)\right|=\gamma-\delta$. The inequality (4.20) implies that at $t=t^{*}$,

$$
P\left(t^{*}\right) \leqq P(0) \exp \left(-\alpha t^{*} / \beta\right)<P(0) \leqq(\gamma-\delta)^{4} / 2 .
$$


However, (4.10a) implies that

$$
\frac{1}{2} S_{1}\left(t^{*}\right)^{4} \leqq P\left(t^{*}\right)
$$

so that

$$
\left|S_{1}\left(t^{*}\right)\right|<\gamma-\delta .
$$

This contradiction shows that $\left|S_{1}(t)\right|<\gamma-\delta$ for all $t \geqq 0$, or equivalently (4.20) holds for all $t \geqq 0$. We conclude that $S_{j}(t) \rightarrow 0$ as $t \rightarrow \infty$. The conditions (4.21) can be rewritten as conditions on $w(x, 0)$ and $w_{t}(x, 0)$. However, the expressions are rather complicated and will not be included here. The preceding remarks may be summarized as:

THEOREM (4.1). If $\varepsilon<0$ and the initial data satisfies the conditions (4.21), then $w(x, t) \rightarrow \sqrt{-\varepsilon} \sin x$ as $t \rightarrow \infty$.

In a manner similar to the above, it can be shown that if the initial conditions are sufficiently close to $-\sqrt{-\varepsilon} \sin x$ then $w(x, t) \rightarrow-\sqrt{-\varepsilon} \sin x$ as $t \rightarrow \infty$.

\section{REFERENCES}

1. S. Woinowsky-Krieger, The effect of axial force on the vibration of hinged bars, J. Appl. Mech. 17 (1950), 35-36. MR 11, 558.

2. R. W. Dickey, Free vibrations and dynamic buckling of the extensible beam, J. Math. Anal. Appl. 29 (1970), 443-454. MR 40 \#6831.

3. J. M. Ball, Initial-boundary value problems for an extensible beam, J. Math. Anal. Appl. 42 (1973), 61-90.

4. - Stability theory for an extensible beam (to appear).

5. R. Courant and D. Hilbert, Methoden der Mathematischen Physik. Vol. I, Springer, Berlin, 1931; English transl., Interscience, New York, 1953. MR 16, 426.

6. E. L. Reiss and B. J. Matkowsky, Nonlinear dynamic buckling of a compressed elastic column, Quart. Appl. Math. 29 (1971), 245-260. MR 44 \#3557.

Department of Mathematics, University of Wisconsin, Madison, Wisconsin 53706 